\title{
Prediction of Long Term Residential Natural Gas Consumption Using ANN
}

\section{Mohsen Hajabdollahi*, Mostafa Hosseinzadeh² and M.M Ghanadi Arab ${ }^{3}$}

${ }^{1}$ Department of Computer Engineering, University of Isfahan, Isfahan, Iran

${ }^{2}$ Department of Industrial Engineering, Islamic Azad university, Arak Branch, Iran

${ }^{3}$ Department of Electrical Engineering, Islamic Azad University, Science and Recearcher Branch, Tehran, Iran

\begin{abstract}
Prediction of residential natural gas consumption in 20 next years was performed in this paper. Artificial neural network (ANN) was used to predict the natural gas in the Kerman, the biggest province in the Iran. The Kerman is included with ten important cities where gas consumption was estimated in each city. The minimum temperature in each year, population growth rate and number of each year were considered as three input variables while the gas consumption in residential section was as an output. The ANN was trained and tested using the data from 2000 to 2008 and applied for prediction in 20 next years from 2008-2028. The predicted result show that the total gas consumption in residential section will increases approximately 1.8 time in 20 next years.
\end{abstract}

Keywords: Natural gas; Residential consumption; Prediction; Artificial neural network

\section{Nomenclature}

b bios
$\mathbf{f}$ transfer function
i population growth rate
$\mathbf{n}$ number of year (year)
NGC natural gas consumption (million $\mathrm{m}^{3}$ )
$\mathbf{P}$ population in the current year
$\mathbf{P}_{\mathbf{0}}$ population in $n$ years ago
T minimum temperature in a year $\left({ }^{\circ} \mathrm{C}\right)$
$\mathbf{w}$ weighting coefficient

\section{Introduction}

Natural gas (NG) is the most efficient and clean burning fossil fuel. The demand for natural gas has a significant impact on the national energy production, energy consumption, national economic growth and people's daily life. Natural gas occupies $1 / 4$ in global energy supply [1]. In 2008, natural gas consumption supplied $24.1 \%$ of the total primary energy consumption of the world. Iran, a member of OPEC since 1961, is ranked amongst the world's top holders of proven oil (ranked fifth owning $8.6 \%$ of oil resources in the world) and natural gas reserves (ranked second owning 15\% of natural gas resources in the world). Because of large gas reserves in Iran and advantages of gas versus oil, the energy policy in this country is based on increase of gas/decrease of oil usage in commercial as well as residential sectors. At the first of 2007, National Iranian Gas Company (NIGC) with 440 million cubic meter refining capacity per day, had 25,000 $\mathrm{km}$ power transition lines and $131,320 \mathrm{~km}$ gas supplying network under its coverage and was providing natural gas to more than 11.6 million homes comprising of approximately 49.9 million people [2]. Modeling and predicting of the natural gas consumption play a vital role in developed and developing countries for policy makers and related organizations. Therefore prediction of natural gas consumption reliably and accurately is an essential part of a country's energy policy. In Iran end-use natural gas consumption can be grouped in four main categories including residential, transportation, industrial and power plant. Previous research in the area of natural gas consumption in various situations reveals that different methods have been used to describe and forecast the natural gas consumption. Liu and Lin [3] forecasted residential natural gas consumption in Taiwan by timeseries models. Durmayaz, Kadioglu and Sen described and forecasted residential heating energy requirement and fuel consumption in Istanbul by a degree-hours method. Sarak and Satman [4] used a degree-day method to forecast heating natural gas consumption in a certain areas of Turkey. Siemek et al. [5] used logistic curve interpretation to estimate the natural gas consumption in Poland. Aras and Aras [6] used auto-regression approach to forecast the natural gas demand for residential section in Turkey. Gutierrez et al. [7] presented Gompertz-type innovation diffusion process as a stochastic growth model of natural gas consumption in Spain. They compared their results with those obtained by stochastic logistic innovation model and stochastic lognormal growth model. Fco et al. [8] proposed a demand forecasting approach for the practical operation of a gas system, based on a decomposition approach to produce up to 3 year-ahead daily forecasts of industrial end-use natural gas consumption. Forouzanfar et al. [2] compared the NLP and GA methods for yearly and seasonal consumption from 1995 to 2008 for residential as well as commercial sectors in Iran. Xu and Wang [1] used polynomial curve and moving average combination projection (PCMACP) model to estimate the future natural gas consumption in China from 2009 to 2015. Li and et al. [9] used a comprehensive approach based on Scenario analysis to forecast the growth of China's natural gas consumption from 1997 to 2029. Potocnik et al. [10] predicted future consumption of natural gas in Slovenia in 2005 and 2006 by using a statistics based machine forecasting model. Sabo et al. [11] presented several possible methods for forecasting the hourly natural gas consumption on the basis of the past natural gas consumption data in the area of the city of Osijek (Croatia) from the beginning of the year 2008.

It should be noted that efficient use of energy resources require accurate prediction of future energy demand. Numerous researchers have analyzed various energy issues and focused on developing appropriate energy demand models to reduce forecasting errors [2].

*Corresponding author: Mohsen Hajabdollahi, Computer Engineering Department University of Isfahan, Isfahan, Iran, E-mail: Mohsen.hajabdollahi@gmail.com

Received January 22, 2013; Accepted February 28, 2013; Published March 04, 2013

Citation: Hajabdollahi M, Hosseinzadeh M, Ghanadi Arab MM (2013) Prediction of Long Term Residential Natural Gas Consumption Using ANN. J Appl Mech Eng 2 : 120. doi: $10.4172 / 2168-9873.1000120$

Copyright: @ 2013 Hajabdollahi M, et al. This is an open-access article distributed under the terms of the Creative Commons Attribution License, which permits unrestricted use, distribution, and reproduction in any medium, provided the original author and source are credited. 
In addition, some authors used artificial neural networks (ANN) to predict the energy consumption. Hsu and Chen [12] proposed a hybrid technique that combines residual modification and neural network estimation for regional peak load forecasting of Taiwan. Yalcinoz and Eminoglu [13] presented a neural network model for short term peak load forecasting, short term total load forecasting and medium term monthly load forecasting in power distribution systems. The suitability of the proposed approach is illustrated through an application to real load shapes from the Turkish electricity distribution corporation in Nigde. Sozen et al. [14] forecasted Turkey's Net energy Consumption using artificial neural network. Gonzalez-Romera et al. [15] used neural networks to predict electric energy demand trend and monthly fluctuation. Tso and Yau [16] presented and compared regression analysis, decision tree and neural networks for the prediction of electricity energy consumption. Hamzacebi [17] forecasted Turkey's electricity consumption with neural networks using a time series type network topology. Tripathi, Upadhyay and Singh used generalized regression and probabilistic neural networks for short-term load forecasting in the electricity market. They tested two different ANN models on data from Australia's Victoria market. Kavaklioglu et al. [18] developed ANN to model and predict the electricity consumption of Turkey. Altan Dombayci [19] proposed an artificial neural network model in order to predict hourly heating energy consumption of a model house designed in Denizli which is located in Central Aegean Region of Turkey.

In this paper, an artificial neural network (ANN) model was used in order to forecast the long-term natural gas consumption in residential section in Kerman province which is located in south of Iran.

\section{Artificial Neural Networks}

Artificial Neural Networks (ANNs) are computational models inspired by the architecture of human brain [18]. Neural networks perform well in applications when the functional form is nonlinear. They are especially useful for prediction problems where mathematical formula and prior knowledge on the relationship between inputs and outputs are unknown [16]. ANN is a model that can learn the relationship between input parameters and controllable or uncontrollable variables, and thus, does not need detailed information about the system. One of the most important advantages of ANN is that it has ability to manage large and complex systems that have many parameters related with each other [19]. ANNs are composed of many computing elements, usually denoted as neurons, working in parallel. The elements are connected by synaptic weights, which are allowed to adapt through a learning process [20]. The feed-forward neural networks are the most popular architectures due to their structural flexibility, good representational capabilities and availability of a large number of training algorithm [21,2]. Neuron is the main part of neural network configuration. A neuron with a single R-element input vector is shown in Figure 1. Here the individual element inputs are $I_{1}, I_{2} \ldots \ldots I_{R}$ multiplied by weights $\mathrm{W}_{11}, \mathrm{~W}_{12} \ldots . ., \mathrm{W}_{1 \mathrm{R}}$ and the weighted values are fed to the summing junction. The neuron has a bias $b$, which is summed with the weighted inputs to form the net input $n$. This sum, n, is the argument of the transfer function $\mathrm{F}$ :

$$
\mathrm{a}=\mathrm{F}(\mathrm{n})=\mathrm{F}\left(\mathrm{W}_{11} \mathrm{I}_{1}+\mathrm{W}_{12}+\mathrm{I}_{2}+\ldots . .+\mathrm{W}_{1 \mathrm{R}} \mathrm{I}_{\mathrm{R}}+\mathrm{b}\right)
$$

This network consists of neurons arranged in layers in which every neuron is connected to all neurons of the next layer. Selection of the input-output training data and input vector of the neural network play a crucial role.

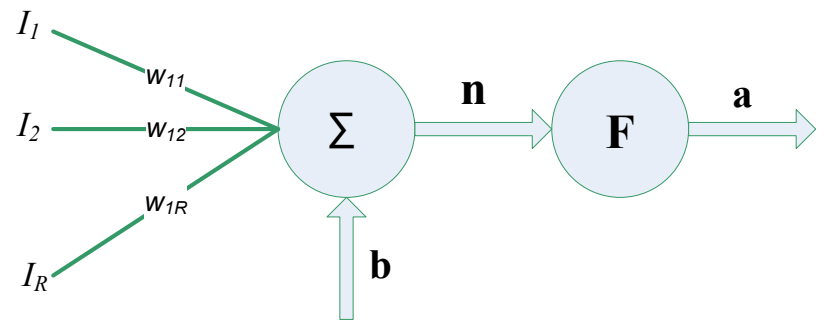

Figure 1: A neuron with a single R-element input vector.

In the training phase of $\mathrm{ANN}$, weights are adjusted in order to produce required outputs with respect to given inputs. Training set is composed of inputs and their respective outputs. In order to train the ANN, all weights and biases should be randomized to input-output pairs selected from the training set and small values. Selected inputs are fed to the network and outputs are calculated and then error value is calculated from the difference between the network's output and the expected output. Repetitions conducted in order to decrease the error to an acceptable value are called training cycles or epochs [19].

\section{Estimation of Natural Gas Consumption in Kerman Province}

Kerman province is located in south of |Iran that is the biggest province. The most important cities in the Kerman are Kerman, Rafsanjan, Zarand, Bam, Baft, Babak, Sirjan, Jiroft, Bardsir and Kahnoj. Artificial neural network was used to estimate the residential natural gas consumption in each city separately. The value of total residential natural gas consumption in Kerman province was estimated by sum of consumption in each city. The essential work in building an ANN natural gas consumption forecasting model is the selection of input variables. Minimum temperature in each year $(T)$, population growth rate $(i)$ and number of each year $(n)$ were selected as input variables while the value of natural gas consumption (NGC) was considered as output. It is worth to mention that the some effective factors such as price of natural gas and consumption culture are taking into account in the number of each year $(n)$.

The data for the residential natural gas consumption in period between 2000 and 2008 were provided from Kerman National Iranian Gas Company (NIGC). Furthermore the data for minimum temperature in each year and population were gathered from Kerman meteorological organization and Kerman management and planning organization, respectively.

The relation between input and output is given as follow where $f$ is a highly non-linear function:

$N G C=f(T, i, n)(2)$

Table 1 presented the population of each city in Kerman province in 1998 and 2008, using this data, the population growth rate can be estimated as:

$$
i=\left(\frac{P}{P_{0}}\right)^{1 / n}-1
$$

where $P$ and $P_{0}$ are the population in the base year and $n$ years later, respectively. The result of population growth rate for each city was shown in table 2 that was assumed to be constant for 20 next years. Furthermore it was assumed that the trend of minimum temperature 
Citation: Hajabdollahi M, Hosseinzadeh M, Ghanadi Arab MM (2013) Prediction of Long Term Residential Natural Gas Consumption Using ANN. J Appl Mech Eng 2: 120. doi:10.4172/2168-9873.1000120

Page 3 of 6

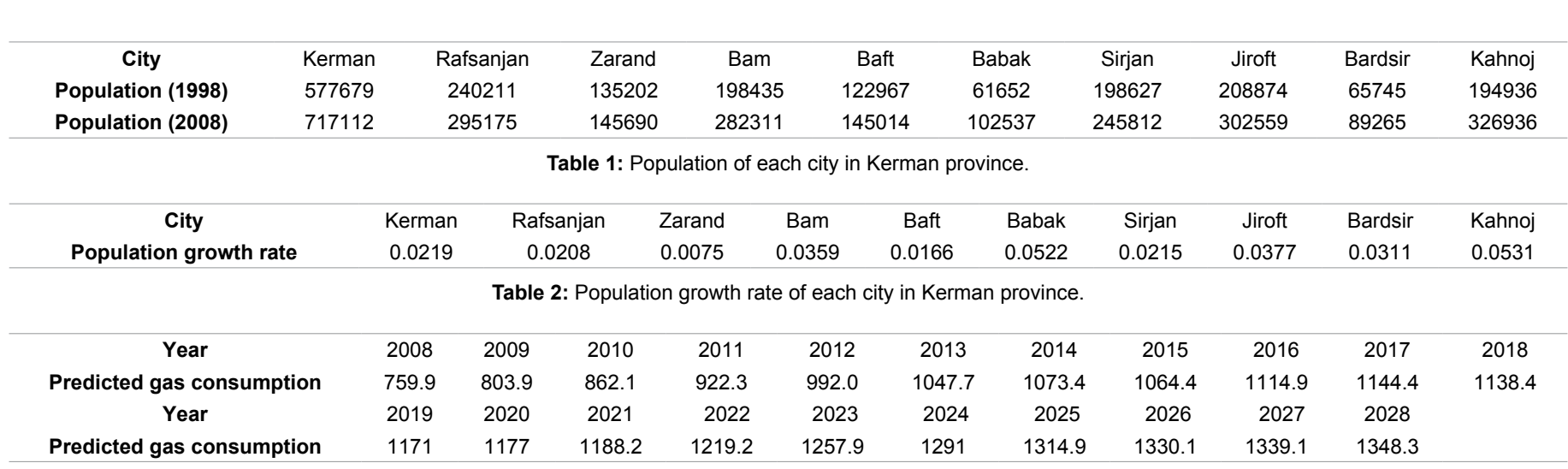

Table 3: Forecasted total natural gas consumption in each year for Kerman province (million cubic meters)

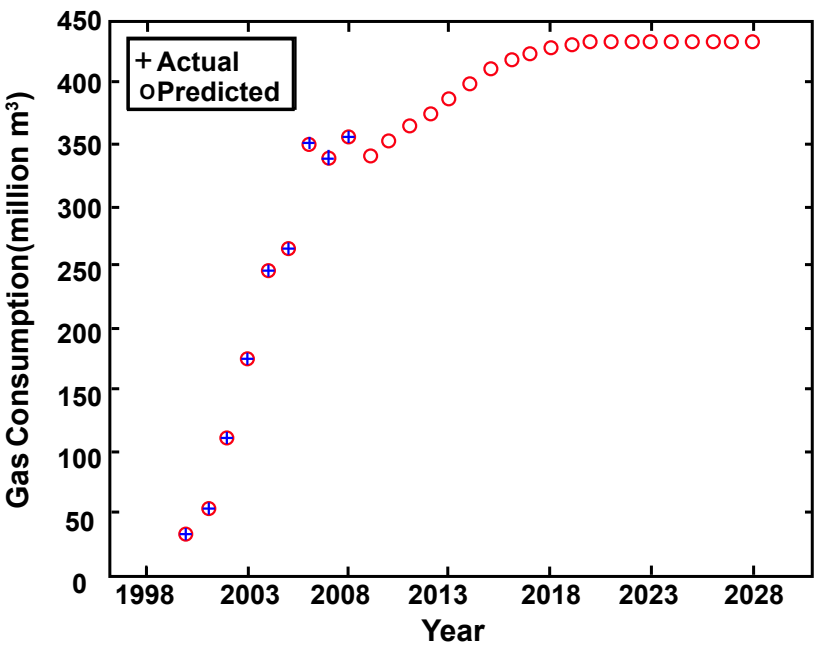

Figure 2: Actual and predicted natural gas consumption for Kerman city.

variation in next 20 years for each city is similar to those in previous 20 years.

To improve the performance of training, all variables were normalized between [0-1] using min-max normalization method:

$$
x=\frac{x_{a c t}-x_{\min }}{x_{\max }-x_{\min }}
$$

This process helps in improving the accuracy of both learning and forecasting modes. To have a good prediction of future natural gas consumption, the neural network by means of two hidden layer with twelve neurons at each layer and an output layout with one neurons, for three input parameters (temperature, number of each year and population growth rate) and one output parameter (gas consumption) were applied using feed forward algorithm. Tan-sigmoid transfer function $\left(-1+\frac{2}{1+e^{-2 n}}\right)$ was used at the hidden layer with $\mathrm{n}$ as the transfer function. Furthermore the linear transfer function $(n)$ was applied at the output layer.

The simulated ANN code was developed in MATLAB software and it was trained and tested using the data from 2000 to 2008 .

The actual and forecasted natural gas consumption in each city is depicted in figures 2-11. The maximum 3 percent error was found between the actual data and predicted results which is acceptable for

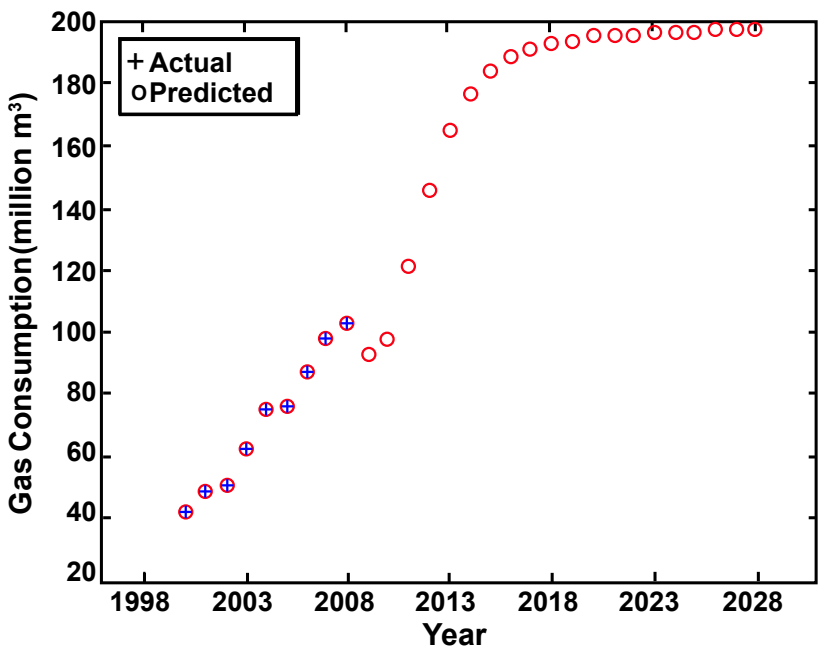

Figure 3: Actual and predicted natural gas consumption for Rafsanjan city.

engineering problem and verified the accuracy of ANN in this case. It is worth to mention that the trend of gas consumption in some cities such as Bam, Kahnoj and Jiroft that does not still have the gas supplying network, were assumed to be such as Rafsanjan due to the similar infrastructures. Actually their neural network model was considered as Rafsanjan model but with their own inputs.

As shown in figures 2-6 the amount of estimated natural gas consumption in some cities such as Kerman, Rafsanjan, Zarand, Baft and Babak will be approximately constant after a specific year due to the fact that gas distribution network will be completed. Moreover one of the effective and important factors for natural gas consumption is minimum temperature that affects the residential natural gas consumption. As it is shown in figures 2, 3, 5, 7, 9, 10 and 11 fluctuation of natural gas consumption in some years is due to the temperature fluctuation. The total predicted natural gas consumption for Kerman province from 2008 to 2028 is evaluated by the sum of predicted consumption for each city given in figure 12 . The results of total forecasted natural gas in residential section are given in table 3 . The results show a rapid growth in natural gas consumption from 2008 to 2013 that is decreased after 2013. It was also observed that the total consumption will be approximately constant after 2028 .

\section{Conclusions}

Modeling and long-term prediction of residential natural gas 


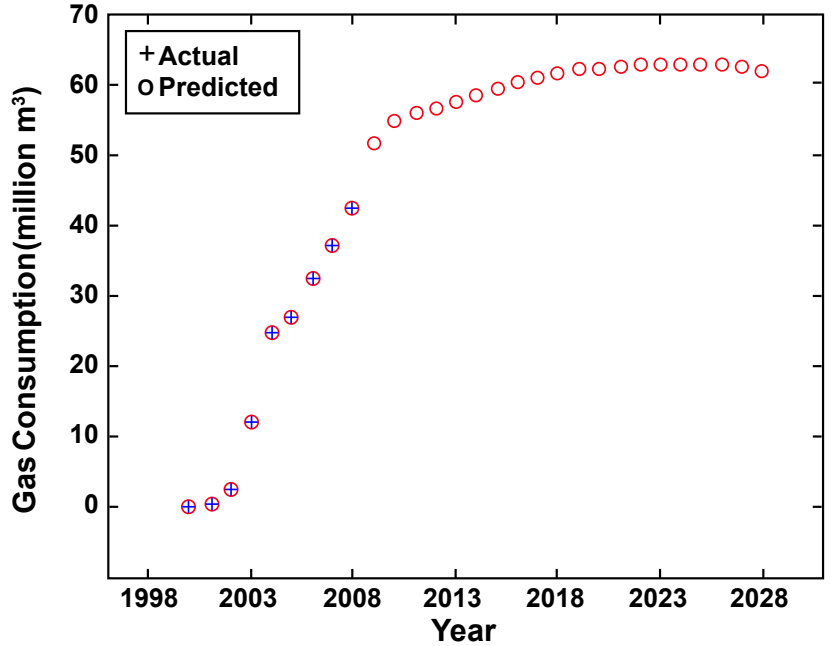

Figure 4: Actual and predicted natural gas consumption for Zarand city.

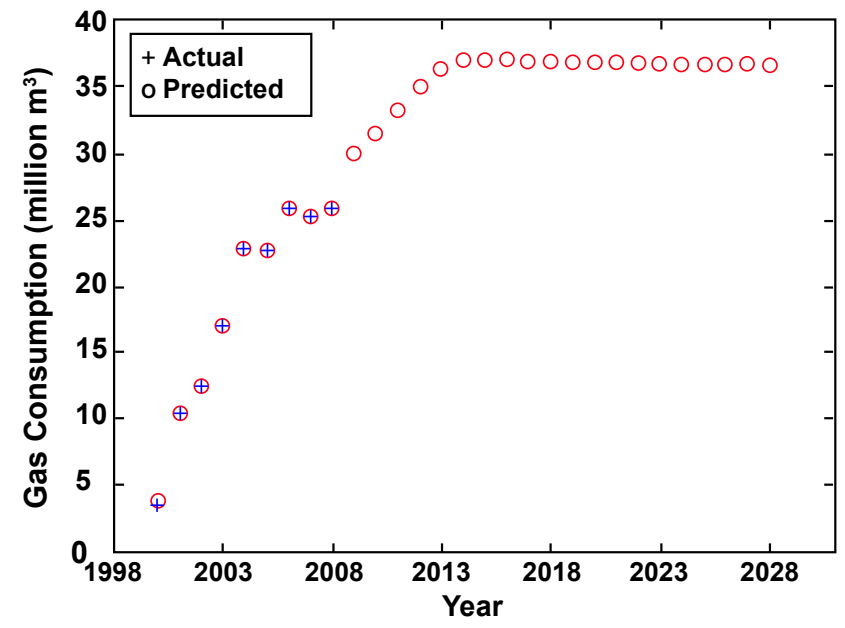

Figure 5: Actual and predicted natural gas consumption for Baft city.

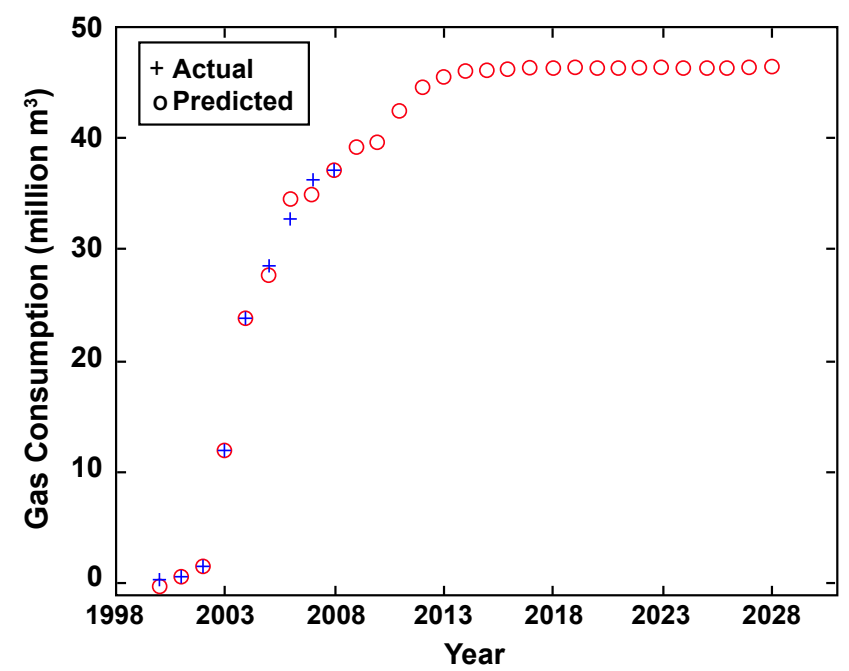

Figure 6: Actual and predicted natural gas consumption for Sahr-e-babak city.

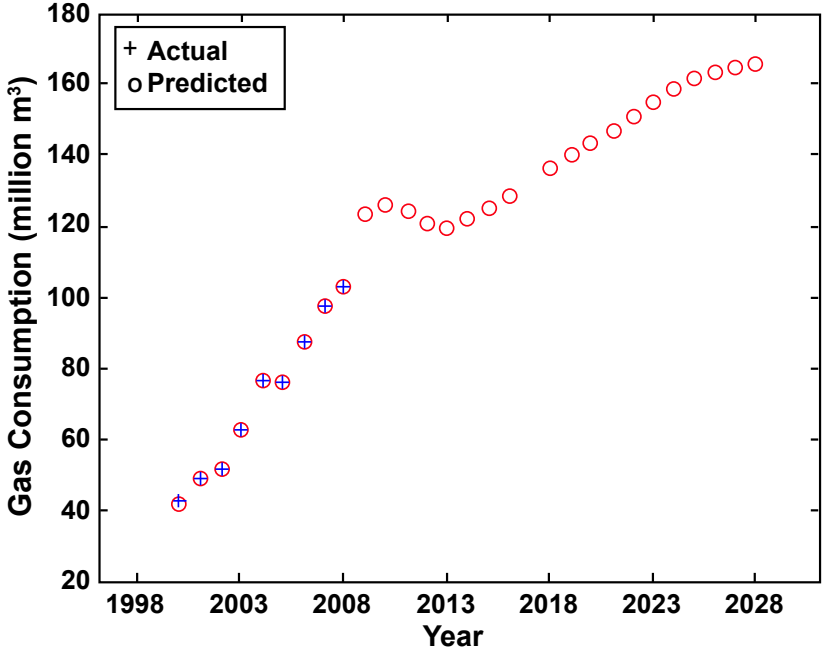

Figure 7: Actual and predicted natural gas consumption for Sirjan city.

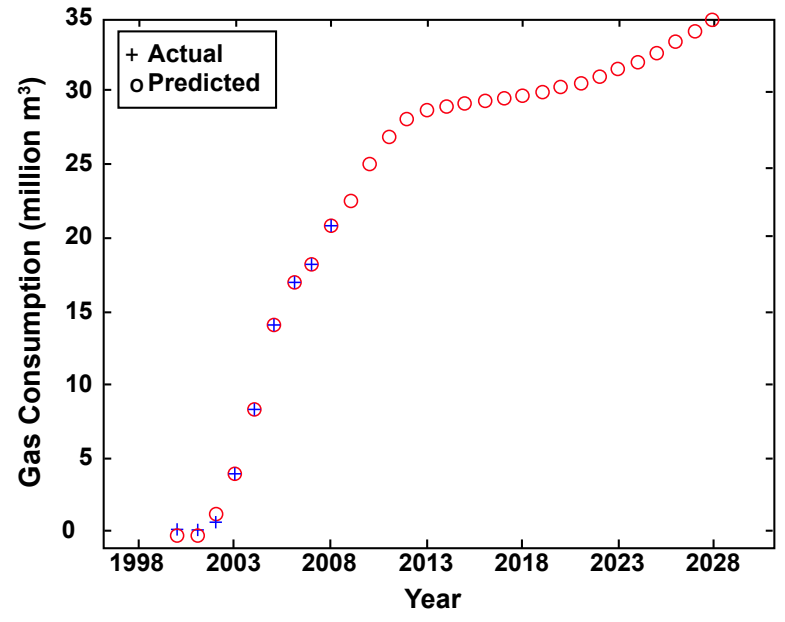

Figure 8: Actual and predicted natural gas consumption for Bardsir city.

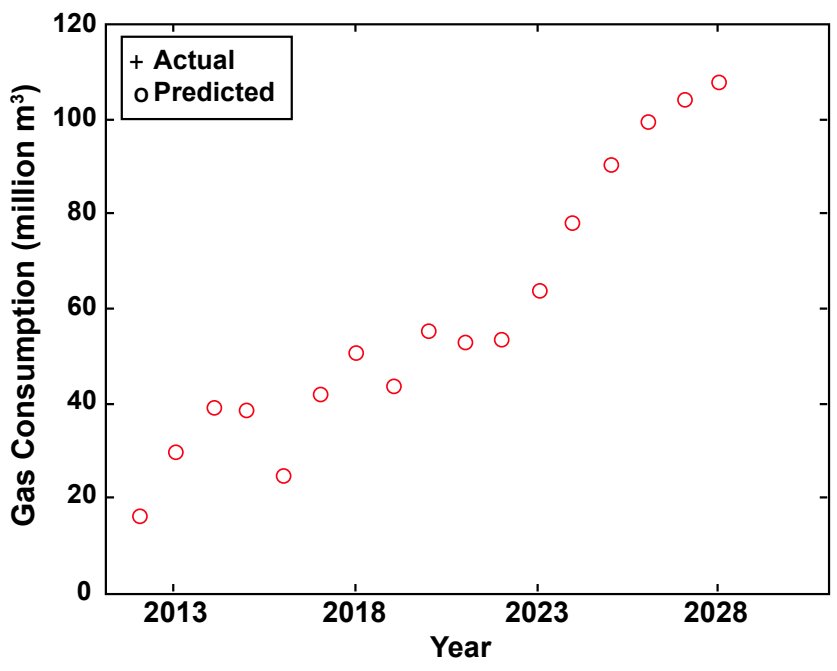

Figure 9: Predicted natural gas consumption for Jiroft city. 
Citation: Hajabdollahi M, Hosseinzadeh M, Ghanadi Arab MM (2013) Prediction of Long Term Residential Natural Gas Consumption Using ANN. J Appl Mech Eng 2: 120. doi:10.4172/2168-9873.1000120

Page 5 of 6

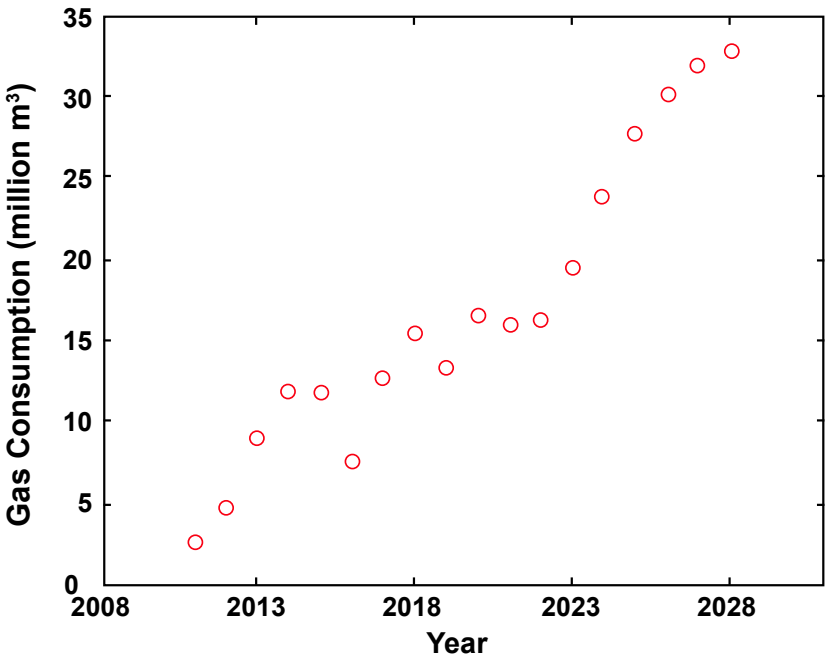

Figure 10: Predicted natural gas consumption for Kahnoj city.

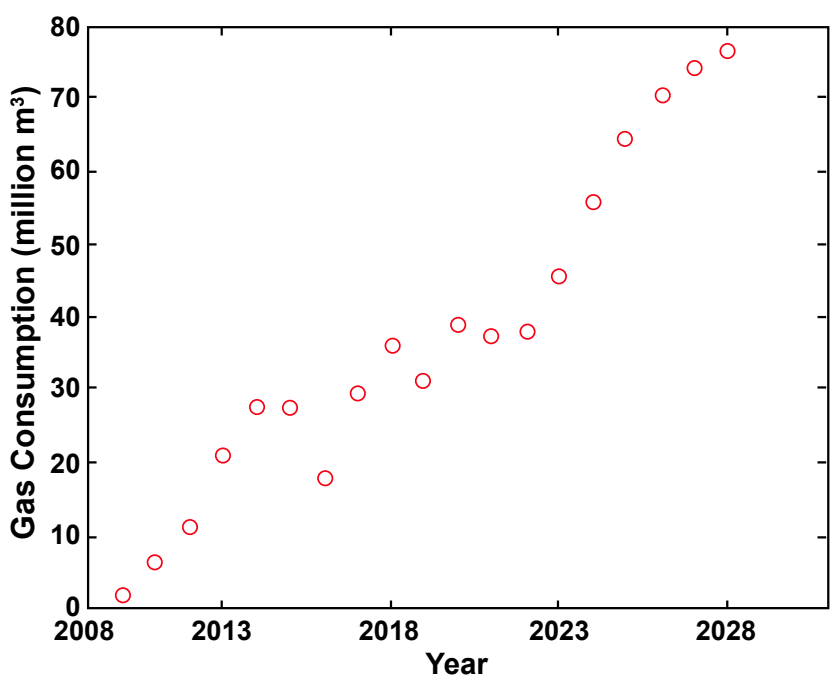

Figure 11: Predicted natural gas consumption for Bam city.

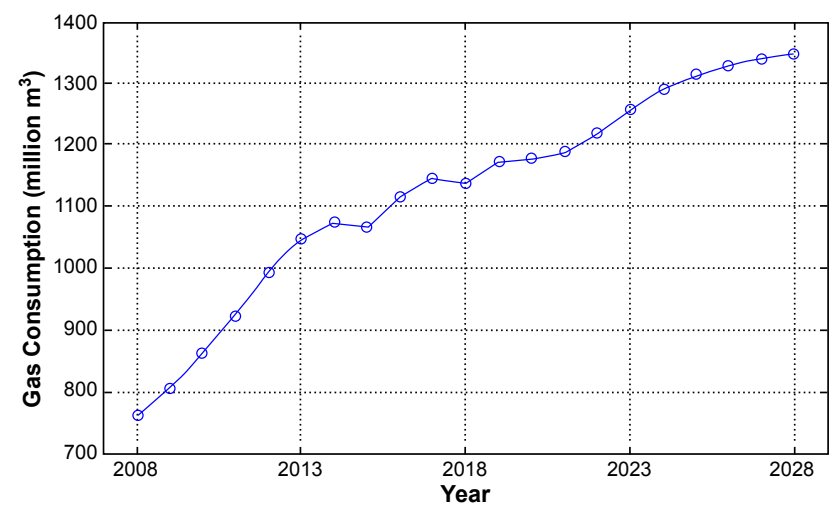

Figure 12: Total predicted natural gas consumption of Kerman province from 2008 to 2028 . consumption in Kerman province (in Iran) was performed in this study. The artificial neural network (ANN) was used to predict the gas consumption in the next 20 years. Three variables including minimum temperature of each year, population growth rate as well as number of each year was considered as input data while residential gas consumption as output. The predicted results were obtained for ten cities in the province using ANN. Finally the results show the approximately 1.8 time increases in the total natural gas consumption in the next 20 years. The maximum 3 percent error between actual data and predicted results show the accuracy of the proposed ANN model for forecasting the natural gas consumption. Therefore ANN is an excellent tool and very effective method for long term prediction in helping the policy makers in the government to make the best decision.

\section{References}

1. Xu G, Wang W (2010) Forecasting China's natural gas consumption based on a combination model. J Nat Gas Chem 19: 493-496.

2. Forouzanfar M, Doustmohammadi A, Menhaj MB, Hasanzadeh S (2010) Modeling and estimation of the natural gas consumption for residential and commercial sectors in Iran. App Enr 87: 268-274.

3. Liu LM, Lin MW (1991) Forecasting residential consumption of natural gas using monthly and quarterly time series. Int J Forecast 7: 3-16.

4. Sarak H, Satman A (2003) The degree-day method to estimate the residential heating natural gas consumption in Turkey: a case study. Enr 28: 929-939.

5. Siemek J, Nagy S, Rychliki S (2003) Estimation of natural-gas consumption in Poland based on the logistic-curve interpretation. Appl Enrgy 75: 1-7.

6. Aras H, Aras N (2004) Forecasting residential natural gas demand. Ene Sou 26: 463-472.

7. Gutierrez R Nafidi A, Gutierrez SR (2005) Forecasting total natural-gas consumption in Spain by using the stochastic Gompertz innovation diffusion model. App Ene 80: 115-124

8. Fco E, Ubeda S, Berzosa A (2007) Modeling and forecasting industrial end-use natural gas consumption. Ene Eco 29: 710-742.

9. Li J, Dong X, Shangguan J, Hook M (2011) Forecasting the growth of China's natural gas consumption. Ene 36:1380-1385.

10. Potocnik P Thaler M, Govekar E, Grabec I, Poredos A (2007) Forecasting risks of natural gas consumption in Slovenia. Ene Poli 35: 4271-4282.

11. Sabo K, Scitovski R, Vazler I, Zekic-Susac M (2011) Mathematical models of natural gas consumption. Ene Con Manag 52: 1721-1727.

12. Hsu CC, Chen CY (2003) Applications of improved grey prediction model for power demand forecasting. Ene Con Manag 44: 2241-2249.

13. Yalcinoz T, Eminoglu U (2005) Short term and medium term power distribution load forecasting by neural networks. Ene Con Manag 46: 1393-1405.

14. Sozen A, Arcaklioglu E, Ozkaymak M (2005) Modelling of the Turkey's net energy consumption using artificial neural network. Int J Com App Tech 22 130-136.

15. Gonzalez-Romera E, Jaramillo-Moran MA, Carmona Fernandez D (2007) Forecasting of the electric energy demand trend and monthly fluctuation with neural networks. Com Ind Eng 52: 336-343.

16. Tso GKF, Yau KKW (2007) Predicting electricity energy consumption: A comparison of regression analysis, Decision tree and neural networks. Ene 32: $1761-1768$

17. Hamzacebi C (2007) Forecasting of Turkey's net electricity energy consumption on sectoral bases. Ene Poli 35: 2009-2016.

18. Kavaklioglu K, Ceylan H, Ozturk HK, Canyurt OE (2009) Modeling and prediction of Turkey's electricity consumption using Artificial Neural Networks. Ene Con Manag 50: 2719-2727.

19. Altan Dombayci O (2010) The prediction of heating energy consumption in a model house by using artificial neural networks in Denizli-Turkey. Adv Eng Soft 41: 141-147. 
Citation: Hajabdollahi M, Hosseinzadeh M, Ghanadi Arab MM (2013) Prediction of Long Term Residential Natural Gas Consumption Using ANN. J Appl Mech Eng 2: 120. doi:10.4172/2168-9873.1000120

Page 6 of 6

20. Tripathi MM, Upadhyay KG, Singh SN (2008) Short-Term Load Forecasting Using Generalized Regression and Probabilistic Neural Networks in the Electricity Market. Elec J 21: 24-34.
21. Sanaye $S$, Hajabdollahi $H$ (2010) Thermal-economic multi-objective optimization of plate fin heat exchanger using genetic algorithm. App Ene 87: 1893-1902. 